\title{
Equilibrium points in the generalised photogravitational non-planar restricted three body problem
}

\author{
Shankaran $^{1}$, J.P.Sharma ${ }^{2}$ and B.Ishwar ${ }^{3^{*}}$ \\ 1. JRF, D.S.T. Project \\ 2. Co-P.I., D.S.T. Project \\ 3. P.I., D.S.T. Project \\ University Department of Mathematics, B.R.A. Bihar University, Muzaffarpur-842001, INDIA \\ "Corresponding Author: e-mail: ishwar_bhola@hotmail.com
}

\begin{abstract}
We generalised the photogravitational non-planar restricted three body problem by considering the smaller primary as an oblate spheroid. With both the primaries radiating, we located the equilibrium points which lie outside the orbital plane, contrary to the classical case. Besides finding the equations of motion of the problem, two equilibrium points lie outside the plane, different from five such points in the classical case.
\end{abstract}

Keywords: Equilibrium Points/ Photogravitational / Non-planar / RTBP.

\section{Introduction}

Poynting (1903) has pointed out that particles, such as small meteors or cosmic dust are comparably affected by gravitation and light radiation force as they approach luminous celestial bodies. In the photogravitational restricted three body problem there exist equilibrium points which, in addition to the five coplanar such points of the classical problem, lie out of the orbital plane. Radzievsky (1950) pointed out the existence of these points. He studied the cases of Sun-planet-particle. Chernikov (1970) and Schuerman (1980) have studied the existence of equilibrium points of the third particle under the influence of gravitation and the radiation forces. The stability of these points was studied in the solar problem by Perezhogin (1976) and by the same author (1982) in the whole range of existence when the smaller body is considered non-luminous. Simmons et.al. (1985) proved the existence of two more out of plane equilibrium points $\mathrm{L}_{8}$ and $\mathrm{L}_{9}$, when bodies are luminous and certain relations between the mass and light parameters hold. They also examined the stability for $\mu=0.5$ and $\mu=0.2$. The out of plane equilibrium points have also been presented as saddle points of the zero velocity surfaces by Ragos and Zagouras (1988a). They discussed the regions of stability for these points of the photogravitational restricted three body problems (1988b). This model has many applications especially in the dynamic behaviour of small objects such as cosmic dust, grains etc. Also it has interesting applications for artificial satellites, future space colonizations or even for sailing vehicles, and spacecraft parking, Friedman (1976). Sharma and Subba Rao (1976) discussed the three dimensional restricted three body problem with oblateness. Douskos and Markellos (2006) found the existence of non-planar equilibrium points in the three dimensional restricted three body problem with oblateness. We were prompted to examine the generalised photogravitational non- planar restricted three body problem in which smaller primary is an oblate spheroid and primaries are radiating. Besides establishing equations of motion of the problem, we locate the equilibrium points

\section{Equations of motion}

We consider three bodies of masses $m_{1}, m_{2}$ and $m$ such that $m_{1}>m_{2}$ and $m$ is an infinitesimal mass. The two primaries $m_{1}$ and $\mathrm{m}_{2}$ are sources of radiation. $\mathrm{q}_{1}$ and $\mathrm{q}_{2}$ are factors characterizing the radiation effects of the two primaries respectively. We assume that $m_{2}$ is an oblate spheroid. The effect of oblateness is denoted by the factor $A_{2}$. Let $r_{i}(i=1,2)$ be the distances between the centre of mass of the bodies $\mathrm{m}_{1}$ and $\mathrm{m}_{2}$ and the centre of mass of body $\mathrm{m}$. 
The force of radiation is given by

$$
\begin{aligned}
& \mathrm{F}=\mathrm{F}_{\mathrm{g}}-\mathrm{F}_{\mathrm{p}}=\mathrm{F}_{\mathrm{g}}\left(1-\frac{\mathrm{F}_{\mathrm{p}}}{\mathrm{F}_{\mathrm{g}}}\right)=(1-\alpha) \mathrm{F}_{\mathrm{g}}=\mathrm{q} \cdot \mathrm{F}_{\mathrm{g}} \\
& \text { where } \alpha=\frac{\mathrm{F}_{\mathrm{p}}}{\mathrm{F}_{\mathrm{g}}} \text { and } \mathrm{q}=1-\alpha
\end{aligned}
$$

Here $F_{g}$ is the gravitational attraction force

$\mathrm{F}_{\mathrm{p}}$ is the radiation pressure force.

Now, the unit of mass is so chosen that the sum of the masses of finite bodies is unity. We suppose that $\mathrm{m}_{1}=1-\mu$ and $\mathrm{m}_{2}=\mu$ where $\mu$ is the ratio of the mass of the smaller primary to the total mass of the primaries and $0<\mu \leq \frac{1}{2}$.

The unit of distance is taken as the distance between the primaries. The unit of time is so chosen that the gravitational constant is unity. Now, let the origin be the barycentre of mass $m_{1}$ at $(-a, 0,0)$ and $m_{2}$ at $(b, 0,0)$.

Hence,

$\mu b+(1-\mu)(-a)=0$,i.e. $\mu(a+b)=a$

Therefore $a=\mu$ and $b=1-\mu$, since $a+b=1$. Thus the co-ordinates of the two masses $(1-\mu)$ and $\mu$ are $(-\mu, 0,0)$ and $(1-\mu, 0,0)$ respectively.

The three dimensional restricted three body problem with an oblate primary $\mathrm{m}_{2}$ and both primaries radiating may be written in barycentric rotating co-ordinate system by the equations of motion

$$
\begin{aligned}
& \ddot{x}-2 n \dot{y}=\frac{\partial \Omega}{\partial x} \\
& \ddot{y}+2 n \dot{x}=\frac{\partial \Omega}{\partial y} \\
& \ddot{z} \quad=\frac{\partial \Omega}{\partial z} \\
& \Omega=\frac{n^{2}}{2}\left(x^{2}+y^{2}\right)+\frac{q_{1}(1-\mu)}{r_{1}}+\frac{q_{2} \mu}{r_{2}}\left[1+\frac{A_{2}}{2 r_{2}^{2}}\left(1-\frac{3 z^{2}}{r_{2}^{2}}\right)\right],
\end{aligned}
$$

Douskos and Markellos (2006).

$\mathrm{n}^{2}=1+\frac{3}{2} \mathrm{~A}_{2}$ is the angular $\mathrm{v}$ elocity of the rotating coordinate system and $\mathrm{A}_{2}$ is the

oblateness co - efficient.

$$
\mathrm{A}_{2}=\frac{\mathrm{AE}^{2}-\mathrm{AP}^{2}}{5 \mathrm{R}^{2}}
$$

where $\mathrm{AE}$ is the equatorial radius

$$
\mathrm{AP} \text { is the polar radius }
$$

and $\mathrm{R}$ is the distance between primaries.

$$
\begin{aligned}
& r_{1}^{2}=(x+\mu)^{2}+y^{2}+z^{2} \\
& r_{2}^{2}=(x-1+\mu)^{2}+y^{2}+z^{2}
\end{aligned}
$$

are the distances of infinitesimal mass from the primaries.

The considerable complexity and interest of the problem over the classical treatment of Lagrange points arises physically from the fact that although the gravitational terms in the potential are scaled by radiation pressure, the rotational term is unmodified since the orbit of the finite masses is unaffected by radiation pressure. We neglect the relativistic Poynting-Robertson effect which may be treated as a perturbation, Chernikov (1970).

\section{Location of Equilibrium points}

We now discuss the location of out of orbital plane $(z \neq 0, y=0)$ points. The location of equilibrium points in general is given by 
$\Omega_{\mathrm{x}}=\Omega_{\mathrm{y}}=\Omega_{\mathrm{z}}=0$.

The second equation is satisfied for $\mathrm{y}=0$. We solve the remaining two equations for $\mathrm{y}=0$ and $\mathrm{z} \neq 0$.

Hence,

$$
\begin{aligned}
& \Omega_{\mathrm{x}}=\mathrm{n}^{2} \mathrm{x}-\frac{(1-\mu) \mathrm{q}_{1}(\mathrm{x}+\mu)}{\mathrm{r}_{1}^{3}}-\frac{\mu \mathrm{q}_{2}(\mathrm{x}-1+\mu)}{\mathrm{r}_{2}^{3}}\left(1+\frac{3 \mathrm{~A}_{2}}{2 \mathrm{r}_{2}^{2}}-\frac{15 \mathrm{~A}_{2} \mathrm{z}^{2}}{2 \mathrm{r}_{2}^{4}}\right)=0 \\
& \Omega_{\mathrm{z}}=\frac{(1-\mu) \mathrm{q}_{1}}{\mathrm{r}_{1}^{3}}+\frac{\mu \mathrm{q}_{2}}{\mathrm{r}_{2}^{3}}\left(1+\frac{9 \mathrm{~A}_{2}}{2 \mathrm{r}_{2}^{2}}-\frac{15 \mathrm{~A}_{2} \mathrm{z}^{2}}{2 \mathrm{r}_{2}^{4}}\right)=0
\end{aligned}
$$

The out-of-plane equilibrium points are found to be located in the (x-z) plane, above and below the centre of oblate primary in symmetrical positions with respect to the (x-y) plane.

With the help of Mathematica, the positions of equilibrium points are approximated to third- order terms in $\mathrm{A}_{2}$ by

$$
\begin{gathered}
\mathrm{x}_{0}=-1+\mu+\frac{3 \sqrt{3}\left(1-\mathrm{q}_{1}\right)(1-\mu)}{\mathrm{q}_{2} \mu} \mathrm{A}_{2}^{3 / 2}+\frac{9 \sqrt{3}\left(1+3 \mathrm{q}_{1}\right)(1-\mu)}{2 \mathrm{q}_{2} \mu} \mathrm{A}_{2}^{5 / 2} \\
-\frac{27\left(1-\mathrm{q}_{1}\right)(1-\mu)\left[2+11(1-\mu) \mathrm{q}_{1}\right]}{2 \mathrm{q}_{2}^{2} \mu^{2}} \mathrm{~A}_{2}^{3}+O\left(\mathrm{~A}_{2}^{7 / 2}\right) \\
\mathrm{z}_{0}=\sqrt{3} \sqrt{\mathrm{A}_{2}}-\frac{9 \mathrm{q}_{1}(1-\mu)}{2 \mathrm{q}_{2} \mu} \mathrm{A}_{2}^{2}-\frac{63 \sqrt{3}\left(1-\mathrm{q}_{1}\right)^{2}(1-\mu)^{2}}{4 \mathrm{q}_{2}^{2} \mu^{2}} \mathrm{~A}_{2}^{5 / 2}+ \\
\frac{81 \mathrm{q}_{1}(1-\mu)}{4 \mathrm{q}_{2} \mu} \mathrm{A}_{2}^{3}+O\left(\mathrm{~A}_{2}^{7 / 2}\right)
\end{gathered}
$$

These equilibrium points exist for $\mathrm{z} \neq 0$ necessarily lying on the $\mathrm{OXZ}$ plane containing the two primaries and the rotation axis. They lie on a plane perpendicular to the orbital one and containing the axis which connects the primaries. They are symmetrical with respect to that axis. Thus new possibilities arise for the existence and stability of equilibrium points since the radiation pressure factors decouple the values of the inverse square law and rotational terms in the potential.

The coordinates of out of plane equilibrium point are depicted in Figure 1 for $A_{2}=0-0.5$ in which panel (I) corresponds to $q_{1}=0.5, q_{2}=1$,(II) $: q_{1}=1, q_{2}=0.5$, (III): $q_{1}=0.5, q_{2}=0.5$ and (IV) $q_{1}=1, q_{2}=1$ (without radiation pressure). It is clear from Figure 1 that when first primary is radiating then the values of both coordinates are negative and increase with $A_{2}$. It is also seen that if the second primary is radiating then the values of coordinates become positive, initially hyperbolic with one arm increasing. Since first primary is bigger than the second primary, therefore radiation effect of first primary is more than that of second one.

\section{Conclusion}

We conclude that out of plane equilibrium points exist in non-planar photogravitational restricted three body problem. This is different from classical restricted three body problem. Stability of these points is an open problem in celestial mechanics. This model may be applied to examine the dynamic behaviour of small objects such as cosmic dust, grains etc.

\section{Acknowledgements}

We are thankful to Dr. K.S.V.S. Narasimhan, Chennai, India and Dr. B.S. Kushvah ISMU, Dhanbad, India for valuable suggestions in preparing this manuscript. We are thankful to D. S. T, Govt. of India, New Delhi for sanctioning the project no. SR /S4/MS: 380/06 dated 13 may 2008. 

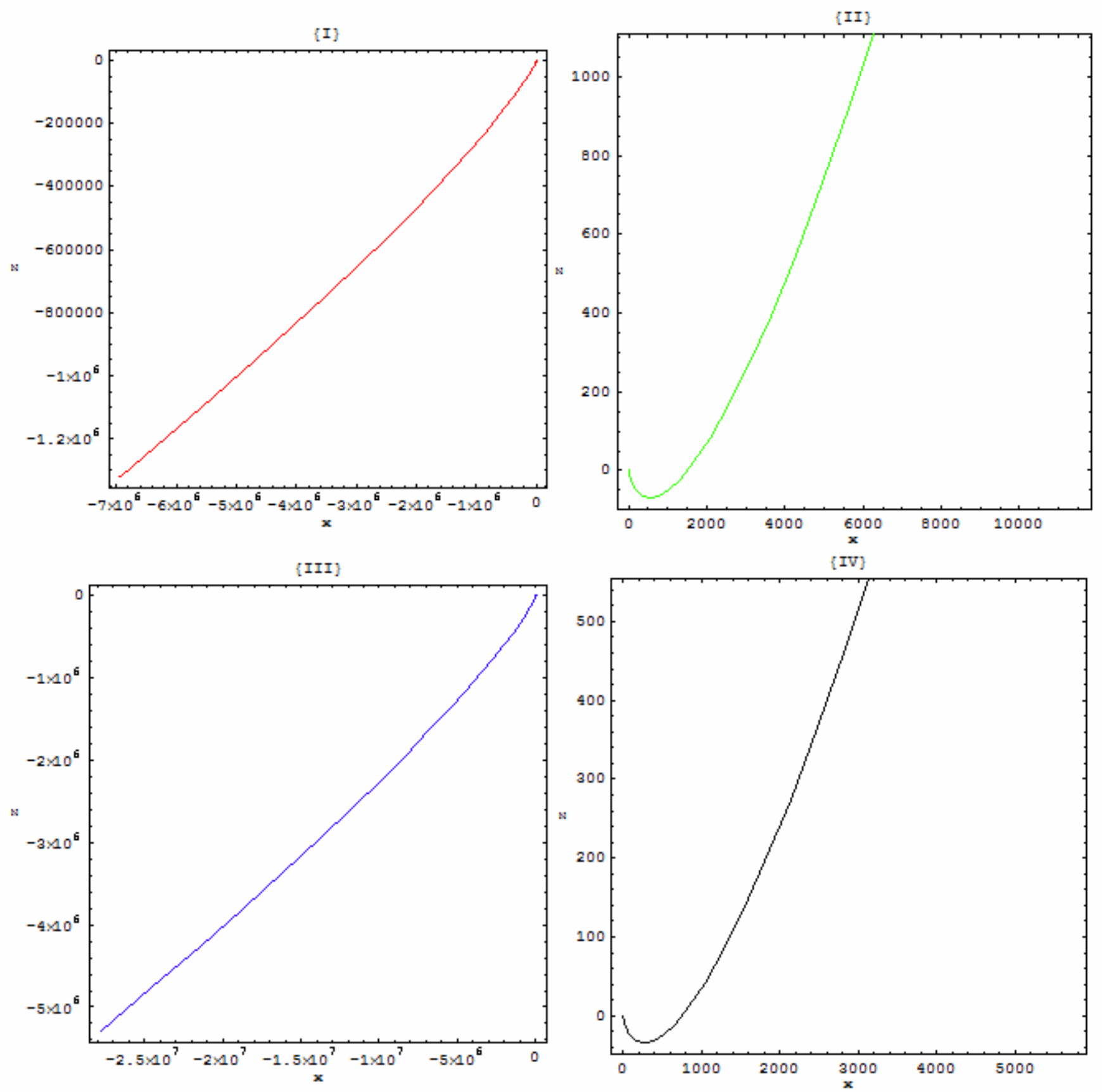

Figure 1. Coordinates of out of plane equilibrium point.

\section{References}

Chernikov,Yu. A. , 1970. The photogravitational restricted three body problem. Soviet Astronomy AJ. vol.14, No.1, pp.176-181.

Douskos, C. N., \& Markellos V.V., 2006. Out-of-plane equilibrium points in the restricted three body problem with oblateness. A \& A, Vol. 446, pp.357-360.

Friedman, L.D., 1976. IN G.A. Gary and K. S. Clifton (eds.), Proceedings of Shuttle-Based Company Science Workshop, George C. Marshall.

Perezhogin, A. A., 1976. Stability of the sixth and seventh libration points in the photogravitational restricted circular three-body problem. Sov. Astron. Letters, Vol.2, pp.174-175. 
Perezhogin, A.A., 1982. The stability of libration points in the restricted photogravitational circular three-body problem. Academy of Science, U.S.S.R. 20, Moscow.

Poynting, J.H., 1903. Philosophical Transactions of the Royal Society of London Series A, Containing papers of a Mathematical or Physical Character. Vol. 202, pp.525-552.

Radzievsky, V.V., 1950. The restricted problem of three bodies taking account of light pressure, Astron Zh, vol.27, pp.250.

Ragos, O., Zagouras, C., 1988a. The zero velocity surfaces in the photogravitational restricted three body problem. Earth, Moon and Planets, Vol.41, pp. 257-278.

Ragos, O., Zagouras, C., 1988b. Periodic solutions about the out of plane equilibrium points in the photogravitational restricted three body problem. Celes.Mech.,vol.44, pp.135.

Schuerman, D.W., 1980. The restricted three body problem including radiation pressure. Astrophys. J., Vol.238, pp.337.

Sharma, R.K., \& Subba Rao, P.V.,1976.Stationary solutions and their characteristic exponents in the restricted three-body problem when the more massive primary is an oblate spheroid. Celes. Mech., vol.13, pp.137.

Simmons, J.F.L., Mcdonald, A.J.C. and Brown, J.C., 1985. The 3-body problem with radiation pressure. Celes. Mech.,vol.35, pp. 145-187.

\section{Biographical notes}

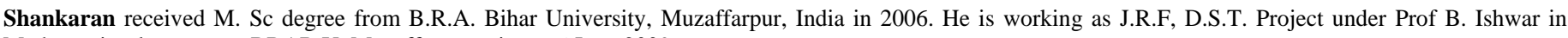
Mathematics department, BRAB.U. Muzaffarpur since 6 June 2009.

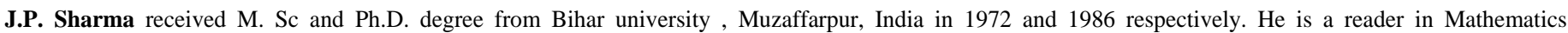
department, BRAB.U. Muzaffarpur, India. His research interests include Celestial Mechanics, Numerical analysis and Fluid mechanics.

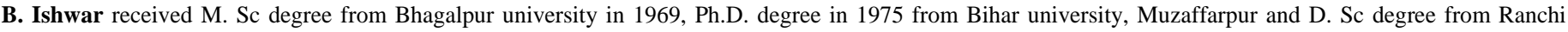

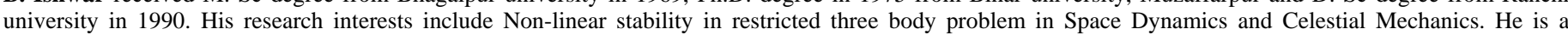

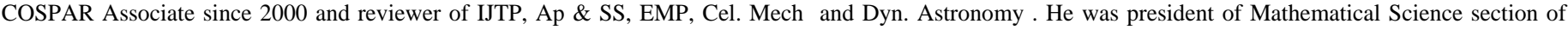
Indian Science Congress association in 2006.

Received September 2010

Accepted February 2011

Final acceptance in revised form March 2011 